\title{
A novel hyperbranched polyester based on 2,2-bis(hydro xylmethyl)butyric acid: synthesis and characterization
}

\author{
Michelina Soccio, ${ }^{1}$ Lara Finelli, ${ }^{1}$ Nadia Lotti, ${ }^{1}$ Paola Marchese,${ }^{1}$ Valentina Siracusa, ${ }^{2}$ \\ Andrea Munari* ${ }^{1 *}$ \\ ${ }^{1}$ Dipartimento di Chimica Applicata e Scienza dei Materiali, Università di Bologna, \\ Viale Risorgimento 2, 40136 Bologna, Italy; Fax ++390512093220; \\ andrea.munari@mail.ing.unibo.it \\ 2 Dipartimento di Metodologie Fisiche e Chimiche per I'Ingegneria, Università di \\ Catania, Viale A. Doria 6, 95125 Catania, Italy
}

(Received: 11 October, 2006; published:16 May, 2007)

\begin{abstract}
A novel hyperbranched polyester was synthesized in bulk from 2,2bis(hydroxymethyl)butyric acid and two different core molecules (1,1,1-tris(hydroxylmethyl)propane and 1,3,5-tris(2-hydroxyethyl)cyanuric acid), using ptoluensolfonic acid, scandium trifluoromethanesulfonate, titanium tetrabutoxide and dibutyltin oxide as catalysts and varied reaction temperature and time. At room temperature the samples obtained appeared as glassy, slightly yellow solids, with $M_{n}$ (calculated by ${ }^{1} \mathrm{H}-\mathrm{NMR}$ spectra) in the range $500-2000$. The degree of branching of the polyesters was determined by ${ }^{13} \mathrm{C}-\mathrm{NMR}$ spectroscopy and was found in the range $0.32-0.53$. The introduction of the monomer in various steps seems to influence slightly the degree of branching, but has no effect on the molecular weight of these polymers. The polyesters exhibited a good thermal stability, as revealed by TGA technique, and the glass transition temperature, determined by DSC, was found to depend on the molecular weight and the kind of catalyst employed.
\end{abstract}

\section{Introduction}

In recent years dendritic polymers have been attracting a considerable and ever growing interest because of their unique physical and chemical properties, different from those of their linear analogues (e.g. lower viscosity in solution and in the molten state, high solubility in common solvents, a significant chain-end effect, etc.) [1-3]. Due to these characteristics, they have been successfully applied in adhesives, coatings, thermosetting resin composites and in a large number of other important applications.

As well known, in the class of dendritic polymers both dendrimers and hyperbranched polymers are included. Dendrimers display a regular globular structure, consisting of a core molecule with two or more functional groups surrounded by several layers (usually named generations) of repeating units containing one or more branch points; therefore they are characterized by a large number of functional groups on the external surface. Nevertheless, the syntheses, carried out both by the divergent or convergent approach, are time consuming and expensive, because each generation has to be built step by step. On the contrary, hyperbranched polymers, which may be considered as the irregular analogues of dendrimers, though displaying similar favourable properties, can be prepared in a simpler and much less expensive way, the syntheses being usually carried out with a 
one-pot procedure. Hyperbranched polymers can be prepared in bulk or in solution, starting from an $\mathrm{AB}_{\mathrm{x}}$-type monomer (in many cases in the presence of a $\mathrm{B}_{\mathrm{y}}$ core molecule, with $y \geq 2$ ) and this is the route followed in most cases, in such systems crosslinking not being possible by a theoretical point of view. Syntheses following the $A_{3}+B_{2}$ approach (or similar) have been carried out too, but in this case much attention has to be paid in order to avoid gel point and therefore the reaction conditions have to be strictly controlled.

Due to the wide variety of suitable monomers, many kinds of hyperbranched polymers have been prepared, from polyesters to polyethers, vinyl polymers, polyamides, polycarbonates, polyurethanes, etc. Even though more and more complete characterizations have been performed, some aspects concerning synthesis conditions and polymer structure appear not to be completely clarified.

It has to be emphasized that among hyperbranched polyesters, those based on 2,2bis(hydroxymethyl)propionic acid (bis-MPA) have been widely investigated, chiefly due to the fact that they are used for important applications and are industrially produced by Perstorp under the trade name of Boltorn. Among commercially available monomers of the $\mathrm{AB}_{2}$ type, 2,2-bis(hydroxymethyl)butyric acid (bis-MBA) attracted our attention, its chemical structure differing from that of bis-MPA only for the presence of an additional $\mathrm{CH}_{2}$ group. Nevertheless, up to now no data are reported in the literature on hyperbranched polyesters based only on such a monomer, which is used mainly in biodegradable copolymers with polylactides and polylactones, the syntheses being based both on esterification and ring opening polymerization $[4,5]$.

In this paper we report the results of a study on the synthesis and the characterization of a novel hyperbranched aliphatic polyester based on 2,2bis(hydroxymethyl)butyric acid (an $\mathrm{AB}_{2}$-type monomer). The research was carried out with the aim of studying the effect of catalyst, of the kind of core molecule and the reaction conditions on the chemical and physical properties of the hyperbranched polymer.

\section{Results and discussion}

NMR spectroscopy is a useful technique widely employed in the structural characterization of polymers and for the determination of some important molecular parameters. In the present case ${ }^{1} \mathrm{H}-\mathrm{NMR}$ spectra were used for the determination of the chemical structure of the hyperbranched polymer and the number-average degree of polymerization $(D)$ of the various samples, whereas ${ }^{13} \mathrm{C}-\mathrm{NMR}$ permitted the calculation of the degree of branching (DB).

\section{Chemical structure}

The chemical structures of 2,2-bis(hydroxymethyl)butyric acid (bis-MBA) and of the two core molecules, 1,3,5-tris(2-hydroxyethyl)cyanuric acid (THEIC) and 1,1,1-tris(hydroxymethyl)propane (TMP), are displayed in Figure 1, whereas the structure of corresponding hyperbranched polymer (poly(bis-MBA)) is reported in Figure 2. 
<smiles>CCC(CO)(CO)C(=O)O</smiles>

bis-MBA<smiles>O=c1n(CCO)c(=O)n(CCO)c(=O)n1CCO</smiles>

THEIC<smiles>CCC(CO)(CO)CO</smiles>

TMP

Fig. 1. Chemical structures of bis-MBA, THEIC and TMP.

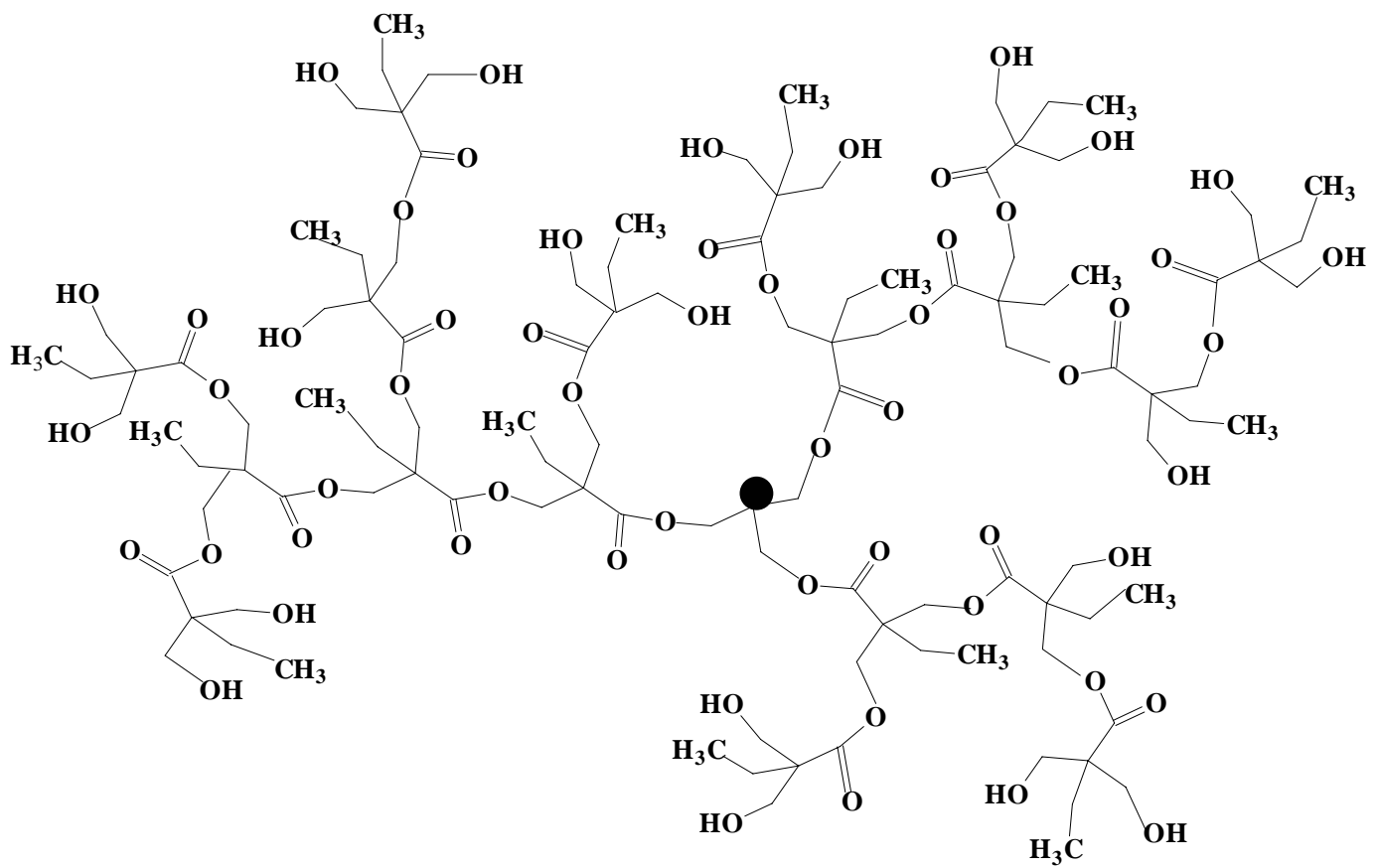

Fig. 2. Chemical structure of hyperbranched poly(bis-MBA); the full point represents the core molecule moiety.

A typical ${ }^{1} \mathrm{H}-\mathrm{NMR}$ spectrum of poly(bis-MBA) is shown in Figure 3 , together with the chemical shift assignments. As can be seen, it is possible to distinguish the protons of the methyl group at $0.77 \mathrm{ppm}$, the methylene protons near to methyl group located at $1.49 \mathrm{ppm}$, whereas the methylene groups in the vicinity of the reacted hydroxyl groups $\left(-\mathrm{CH}_{2} \mathrm{OR}\right)$ and those attached to the unreacted hydroxyl groups $\left(-\mathrm{CH}_{2} \mathrm{OH}\right)$ resonate at $4.17 \mathrm{ppm}$ and $3.50 \mathrm{ppm}$, respectively. It has to be emphasized that the signal of $\mathrm{CH}_{2} \mathrm{OH}$ is not well separated from that of water, which, in the experimental conditions adopted, resonates at $3.32 \mathrm{ppm}$. This situation does not allow a correct determination of the integral signals to be employed in the calculation of $\mathrm{DP}_{\mathrm{n}}$ and for this purpose the analysis conditions were changed, as reported in the following. 


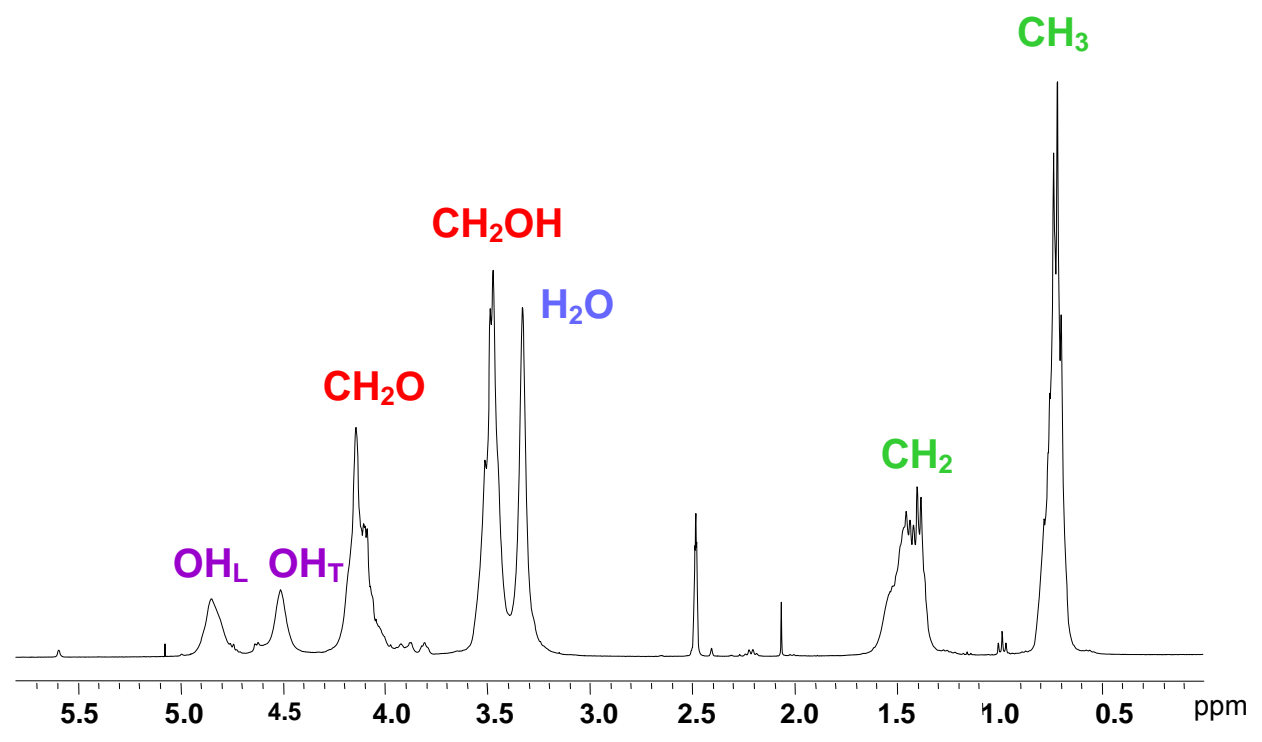

Fig. 3. ${ }^{1} \mathrm{H}-\mathrm{NMR}$ spectrum of poly(bis-MBA) in DMSO- $d_{6}$ (polymer concentration: 3.0 wt \%) at room temperature. The spectrum refers to the sample obtained by run 10 (see Table 2).

All the spectra obtained confirm the expected chemical structure of poly(bis-MBA).

\section{Degree of polymerization}

Difficulties of different kinds often arise in measuring the correct molecular weight of dendritic polymers by gel permeation chromatography. A basic problem is the use of linear polymer samples (commonly polystyrene) as standards, which results in very unrealistic values for the molecular masses of the branched polymers. In addition, highly branched samples could interact strongly with the column stationary phase [6]. In this view, ${ }^{1} \mathrm{H}-\mathrm{NMR}$ spectroscopy represents an important technique for the determination of the number average molecular weight for this kind of polymers.

The number-average degree of polymerization $\left(\mathrm{DP}_{\mathrm{n}}\right)$ of hyperbranched polymers can be expressed by [7]:

$$
D P_{n}=\frac{N(B)+N(A B)-(f-2) N_{\text {core }}}{N(B)-N(A B)-(f-2) N_{\text {core }}}
$$

where in the present case $N(B)$ and $N(A B)$ are the number of unreacted and reacted $-\mathrm{CH}_{2} \mathrm{OH}$ groups respectively, $\mathrm{f}$ is core functionality and $\mathrm{N}_{\text {core }}$ is the number of core molecules. $N(B)$ and $N(A B)$ can be determined from the integrals of the ${ }^{1} \mathrm{H}-\mathrm{NMR}$ signals corresponding to $\mathrm{B}\left(-\mathrm{CH}_{2} \mathrm{OH}\right)$ and $\mathrm{AB}\left(-\mathrm{CH}_{2} \mathrm{OR}\right)$ groups, respectively, whereas $\mathrm{N}_{\text {core }}$ can be calculated from the monomer/core feed ratio.

The ${ }^{1} \mathrm{H}-\mathrm{NMR}$ analyses were performed in $\mathrm{DMSO}-d_{6}$ at room temperature, but the results were not satisfactory, due to the presence of the peak of water, that in some cases superimposes to the signal of $-\mathrm{CH}_{2} \mathrm{OH}$ (see Figure 3). In order to avoid such interference, some attempts were made with the aim of weakening the intermolecular $\mathrm{H}$-bonds between water molecules, moving to higher magnetic field the $\mathrm{H}_{2} \mathrm{O}$ proton signal $[8,9]$. In this view, the following measurement conditions were changed:

i) analysis temperature;

ii) solvent polarity, by adding appropriate salts; 
iii) sample concentration.

As one can see from Figure 4, the spectrum performed at a higher temperature, i.e. at $75{ }^{\circ} \mathrm{C}$, shows the shifting of the signal of hydroxyl groups to higher magnetic fields, but the simultaneous enlargement of such peak does not permit a good separation from that of $-\mathrm{CH}_{2} \mathrm{OH}$.

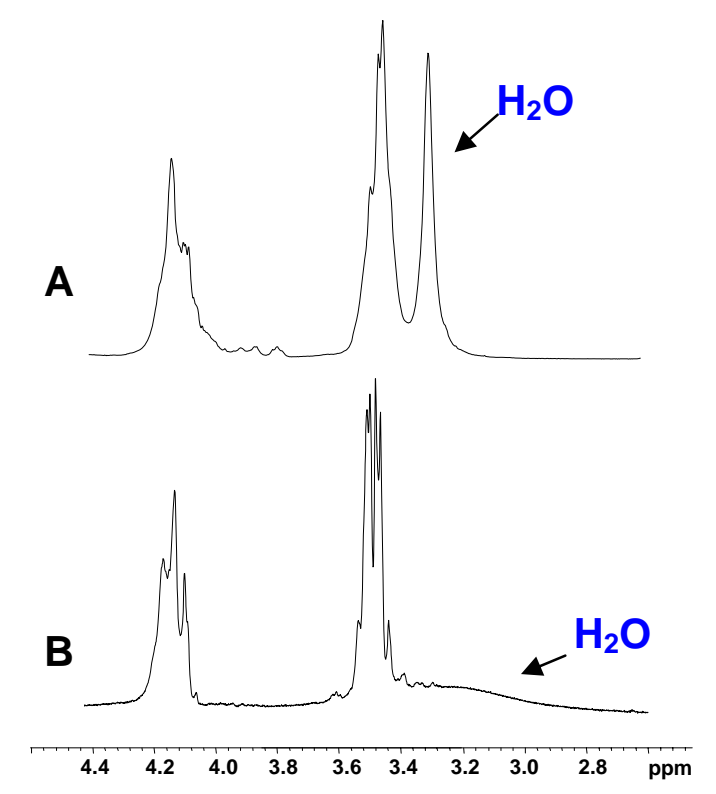

Fig. 4. ${ }^{1} \mathrm{H}-\mathrm{NMR}$ spectra of poly (bis-MBA) in DMSO- $d_{6}$ (polymer concentration of 3.0 wt \%): (A) at room temperature; (B) at $75{ }^{\circ} \mathrm{C}$. The spectrum refers to the sample obtained by run 10 (see Table 2).

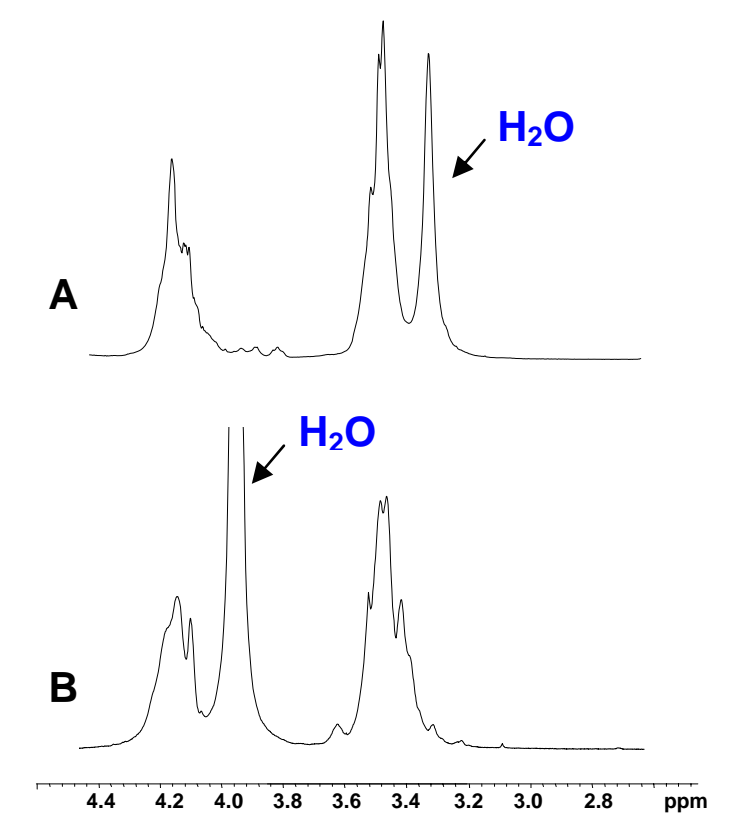

Fig. 5. ${ }^{1} \mathrm{H}-\mathrm{NMR}$ spectra at room temperature of poly (bis-MBA) (polymer concentration of $3.0 \mathrm{wt} \%$ ) in: (A) DMSO- $d_{6}$; (B) $5.0 \% \mathrm{LiCl} /$ DMSO- $d_{6}$. The spectrum refers to the sample obtained by run 10 (see Table 2).

As concerns solvent polarity, different amounts of $\mathrm{LiCl}$ (from 1.7 to $5.0 \mathrm{wt} \%$ ) were added to DMSO- $d_{6}$, but the results were not completely satisfactory. In fact, repeated 
measurements did not show good reproducibility and in addition the water signal often superimposes to other important ones, in particular that of $\mathrm{CH}_{2} \mathrm{OR}$ group (see Figure 5).

On the contrary, good results were obtained by changing sample concentration: in fact, at low concentrations, $\mathrm{CH}_{2} \mathrm{OH}$ and water signals are well resolved, being located at 3.50 and $3.26 \mathrm{ppm}$, respectively (see Figure 6). As a consequence, the $\mathrm{DP}_{\mathrm{n}}$ values were calculated from the spectra obtained using a DMSO- $d_{6}$ solution containing 0.5 wt $\%$ of polymer.

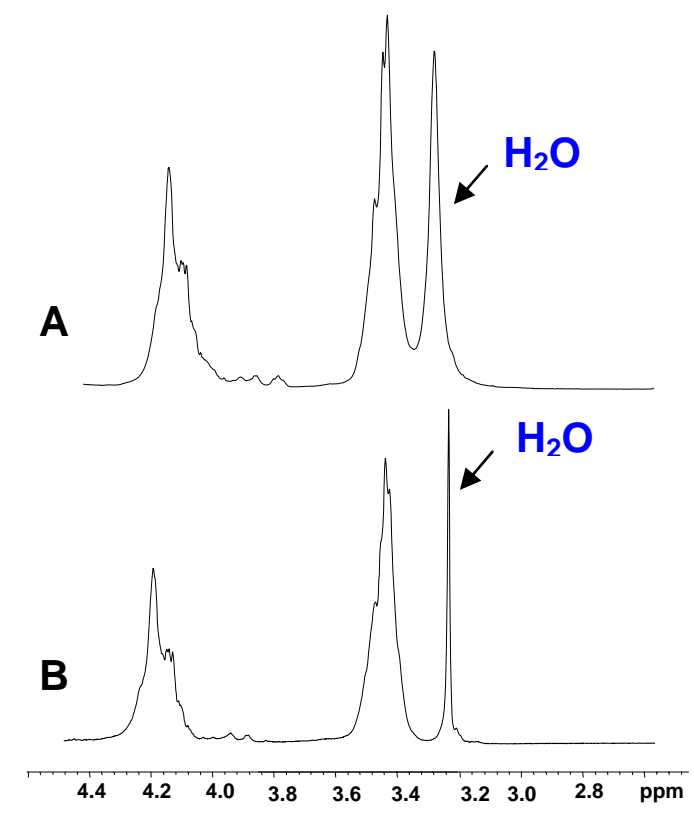

Fig. 6. ${ }^{1} \mathrm{H}-\mathrm{NMR}$ spectra at room temperature of poly (bis-MBA) in DMSO- $d_{6}$ with different polymer concentration: (A) $3.0 \mathrm{wt} \%$; (B) $0.5 \mathrm{wt} \%$. The spectrum refers to the sample obtained by run 10 (see Table 2).

\section{Degree of Branching}

It is well known that a hyperbranched structure contains three different kinds of structural units: dendritic $(\mathrm{D})$, linear $(\mathrm{L})$, and terminal $(\mathrm{T})$. To describe hyperbranched polymers, Fréchet et al. introduced the average degree of branching (DB) [10], defined as the sum of the number of terminal and dendritic units divided by the total sum of all the units:

$$
D B=\frac{N(D)+N(T)}{N(D)+N(L)+N(T)}
$$

For a perfect dendrimer $(\mathrm{N}(\mathrm{L})=0)$ the degree of branching is 1 , whereas for hyperbranched polymers it is less than unity.

In the ${ }^{13} \mathrm{C}$-NMR spectrum, the quarternary carbons of the different repeating units can be well distinguished. In fact, their peaks are characterized by chemical shifts, which depend on the degree of substitution of the repeating unit. Therefore, the relative amounts of $T, L$ and $D$ units can be determined by comparing the integrals of the different peaks. In particular, for poly(bis-MBA), the signals of terminal, linear and dendritic units appeared at $\delta 53.62, \delta 52.00$ and $\delta 50.03$, respectively, as shown in Figure 7 . 


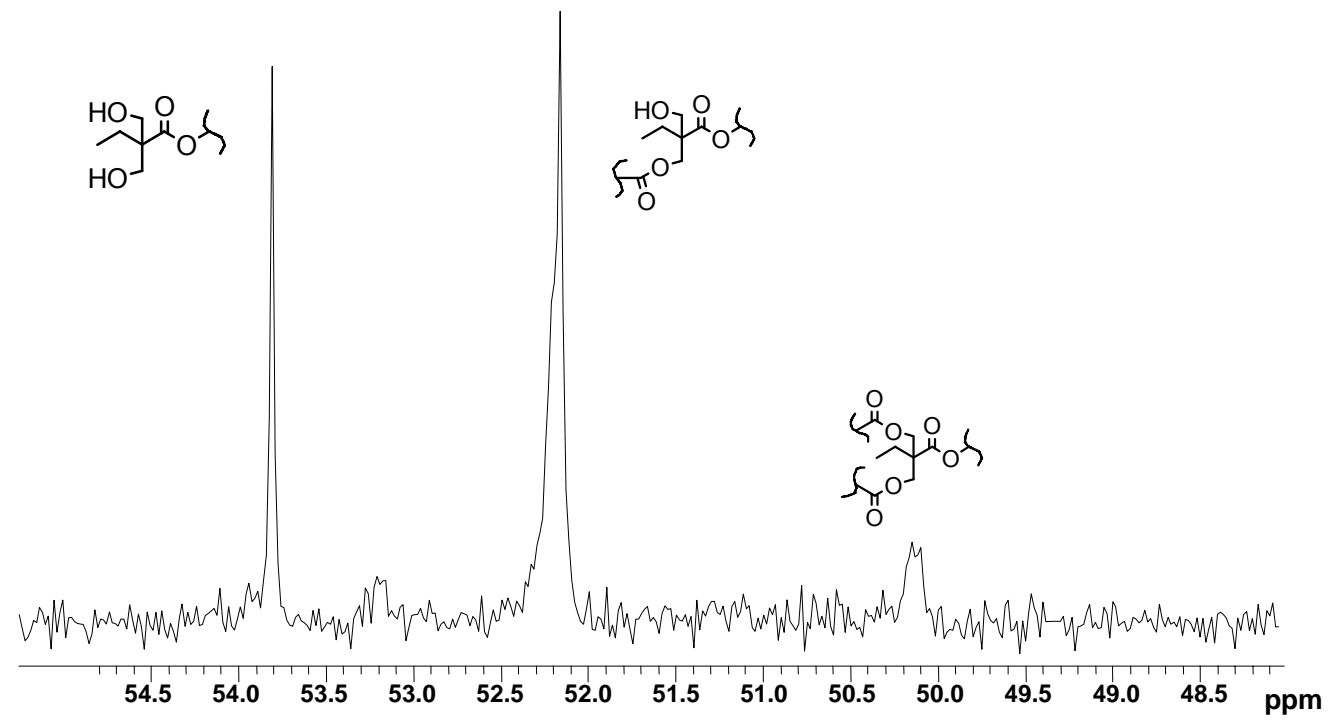

Fig. 7. ${ }^{13} \mathrm{C}-\mathrm{NMR}$ spectrum of poly(bis-MBA). Quaternary carbon region of the spectrum was magnified. The spectrum refers to the sample obtained by run 4 (see Table 2).

The DBs, calculated according to eq. (2), are collected in Tables 1-3 for most of the samples synthesized, together with the number-average molecular weight data $\left(M_{n}\right)$, derived from $\mathrm{DP}_{\mathrm{n}}$ values.

\section{Synthesis of polymer samples}

The synthesis in bulk of hyperbranched polyesters starting from an $A B_{2}$-type monomer is a well known route that in principle should bring to high molecular weight samples, as it happens in the case of a large number of linear and randomly branched polycondensates. Nevertheless, when starting from an $\mathrm{AB}_{2}$-type monomer, the architectural complexity of the resulting polymer leads to some difficulties in obtaining large molecules, mainly due to hindering and substitution effects. These effects prevent some of the terminal groups of the growing molecules from reacting, giving therefore origin to linear (or, better, 'pseudo-linear') branches of the chains. In addition, the role of the catalyst is not yet well clarified, even though in most cases, when starting from monomers with hydroxyl and carboxyl end groups, acids able to catalyze the direct esterification reactions have been successfully employed.

Preliminarily, a series of experiments was carried out, starting from 2,2bis(hydroxymethyl)butiric acid at various temperatures, using some of the catalysts commonly employed in polycondensation, i.e, dibutyltin oxide, titanium tetrabutoxide, scandium triflate, and p-toluene sulfonic acid. The results obtained indicated scandium triflate and $p$-toluene sulfonic acid as the most promising catalysts in the case of the synthesis in bulk of poly(bis-MBA).

As concerns catalyst concentration, another series of tests was performed in the same reaction conditions, but using different amounts of the two preliminarily selected catalysts. From the results, collected in Tab. 1, it appears that ScTF contents above $0.1 \mathrm{~mol} \%$ lead to a rather quick gelation, whereas in the case of pTSA a content of $0.35 \mathrm{~mol} \%$ accelerates sufficiently the polymerization reaction, so that in the further syntheses these concentrations were adopted for the two catalysts, respectively. 
Tab. 1. Effect of catalyst concentration in poly(bis-MBA) synthesis.

\begin{tabular}{|c|c|c|c|c|c|c|c|}
\hline Run & Cat & $\begin{array}{c}\mathrm{mol}_{\mathrm{CAT}} / \mathrm{mol}_{\mathrm{POL}} \\
(\%)\end{array}$ & Core & $\begin{array}{c}\mathrm{T} \\
\left({ }^{\circ} \mathrm{C}\right)\end{array}$ & $\begin{array}{l}\mathrm{t}_{1}{ }^{\mathrm{a}} \\
(\mathrm{h})\end{array}$ & $\begin{array}{l}t_{2}^{b} \\
(h)\end{array}$ & $M_{n}{ }^{c}$ \\
\hline$A$ & TFSc & $0.10 \%$ & TMP & 160 & 2.5 & 1.0 & 1780 \\
\hline $\mathrm{A} 1$ & TFSc & $0.35 \%$ & TMP & 160 & 2.5 & 0.4 & n.d.* \\
\hline $\mathrm{A} 2$ & TFSc & $0.50 \%$ & TMP & 160 & 2.5 & 0.02 & n.d.* \\
\hline$B$ & ATS & $0.10 \%$ & TMP & 160 & 2.5 & 1.0 & 440 \\
\hline B1 & ATS & $0.35 \%$ & TMP & 160 & 2.5 & 1.0 & 590 \\
\hline B2 & ATS & $0.50 \%$ & TMP & 160 & 2.5 & 1.0 & 650 \\
\hline
\end{tabular}

As the introduction of a core molecule favours the structural regularity of the hyperbranched polymer, in most cases the syntheses were performed employing TMP, whose molecule is characterized by three alcoholic functionalities and therefore is of the $\mathrm{B}_{3}$-type, in the molar ratio of $1 / 45$ on respect of bis-MBA (corresponding to a fourth generation hyperbranched polymer). In some cases THEIC (which is another $\mathrm{B}_{3}$-type molecule) instead of TMP was used. The syntheses were carried out in different temperature conditions and for different reaction times, as reported in Tab. 2 , which presents also the values of $M_{n}$ and $D B$ of the samples, obtained by ${ }^{1} \mathrm{H}-\mathrm{NMR}$ and ${ }^{13} \mathrm{C}-\mathrm{NMR}$ spectroscopy, respectively (see the appropriate Sections).

Tab. 2. Synthesis conditions and characterization data for poly(bis-MBA) adopting the one-pot procedure.

\begin{tabular}{|c|c|c|c|c|c|c|c|c|c|c|c|}
\hline Run & Cat & Core & $\begin{array}{c}\mathrm{T} \\
\left({ }^{\circ} \mathrm{C}\right)\end{array}$ & $\begin{array}{l}\mathrm{t}_{1}{ }^{\mathrm{a}} \\
(\mathrm{h})\end{array}$ & $\begin{array}{l}\mathrm{t}_{2}{ }^{\mathrm{b}} \\
\text { (h) }\end{array}$ & $M_{n}{ }^{c}$ & DB & $\begin{array}{c}-\mathrm{COOH} \\
(\mathrm{mmol} / \mathrm{g} \mathrm{pol} .)\end{array}$ & $\begin{array}{c}-\mathrm{OH} \\
(\mathrm{mmol} / \mathrm{g} \mathrm{pol} .)\end{array}$ & $\begin{array}{l}\mathrm{T}_{\text {i.d. }} \\
\left({ }^{\circ} \mathrm{C}\right)\end{array}$ & $\begin{array}{l}\mathrm{T}_{\mathrm{g}} \\
\left({ }^{\circ} \mathrm{C}\right)\end{array}$ \\
\hline 1 & TFSc & TMP & 160 & 2.0 & 0.6 & 760 & 0.35 & 0.56 & 7.0 & 294 & 49 \\
\hline 2 & TFSc & TMP & 160 & 3.5 & 1.0 & 1790 & 0.32 & 0.26 & 6.7 & 293 & 55 \\
\hline 3 & TFSc & TMP & 170 & 2.7 & 0.6 & n.d. ${ }^{*}$ & n.d. ${ }^{*}$ & n.d. ${ }^{*}$ & n.d. ${ }^{*}$ & 307 & 61 \\
\hline 4 & TFSc & TMP & 170 & 3.8 & 0.0 & 1580 & 0.40 & 0.70 & 7.2 & 302 & 55 \\
\hline 5 & ATS & TMP & 190 & 2.5 & 3.5 & 830 & 0.43 & 1.3 & 6.4 & 305 & 41 \\
\hline 6 & ATS & TMP & 190 & 3.0 & 1.5 & 780 & 0.42 & 1.4 & 6.8 & 309 & 37 \\
\hline 7 & ATS & TMP & 190 & 5.5 & 2.0 & 1000 & 0.38 & 1.0 & 5.2 & 308 & 43 \\
\hline 8 & TFSc & THEIC & 160 & 2.0 & 0.6 & 1130 & 0.35 & 0.7 & 6.7 & 294 & 55 \\
\hline 9 & TFSc & THEIC & 160 & 3.5 & 1.0 & n.d. ${ }^{*}$ & n.d. ${ }^{*}$ & n.d. ${ }^{*}$ & n.d. ${ }^{*}$ & 300 & 59 \\
\hline 10 & ATS & THEIC & 190 & 2.7 & 3.8 & 750 & 0.44 & 1.1 & 5.6 & 305 & 39 \\
\hline 11 & ATS & THEIC & 190 & 5.5 & 2.0 & 1200 & 0.40 & 1.0 & 4.8 & 304 & 42 \\
\hline
\end{tabular}

From these results, it appears that gelation, which in principle should be avoided in the polymerization of an $A B_{2}+B_{3}$ system, sometimes occurs (see run 3 , as an example), but only when ScTF is employed. This fact is not completely surprising, as ScTF is a well known transesterification catalyst and therefore we can suppose that 
in our syntheses it favours these kinds of reactions, so that ester links form between growing molecules, in some cases giving rise to a crosslinked system. It has to be emphasized that such catalysts act also on etherification reactions, as reported in the case of the hyperbranched polycondensation of 2,2-bis(hydroxymethyl) propanoic acid [11], favouring the formation of a crosslinked system.

Another remark to be made is that ScTF appears to be efficient at lower temperatures, the polymer requiring a lower time to reach a relatively high value of $M_{n}$. On the other hand, the samples obtained using pTSA show a higher DB and therefore a more regular structure (see run 1 and 6 or run 8 and 11); it has to be taken into account that the comparison has to be made at a fixed molecular weight, as in hyperbranched polymers DB decreases with increasing $M_{n}$ [12]. Lastly, as far as the effect of the core structure is concerned, the use of THEIC instead of TMP did not influence significantly the polymer growth.

Tab. 3. Multi-step syntheses of poly(bis-MBA).

\begin{tabular}{|c|c|c|c|c|c|c|c|c|c|}
\hline Run & Cat & Core & $\begin{array}{c}\mathrm{T} \\
\left({ }^{\circ} \mathrm{C}\right)\end{array}$ & $\begin{array}{l}t_{1}{ }^{a} \\
(h)\end{array}$ & $\begin{array}{l}t_{2}{ }^{b} \\
\text { (h) }\end{array}$ & $M_{n}{ }^{c}$ & DB & $\begin{array}{c}\mathrm{T}_{\mathrm{id}} \\
\left({ }^{\circ} \mathrm{C}\right)\end{array}$ & $\begin{array}{c}\mathrm{T}_{\mathrm{g}} \\
\left({ }^{\circ} \mathrm{C}\right)\end{array}$ \\
\hline & & & 100 & 3.5 & 1.0 & & & & \\
\hline \multirow[t]{3}{*}{12} & ATS & TMP & 100 & 3.5 & 0.5 & 470 & 0.52 & 296 & 24 \\
\hline & & & 130 & 5.0 & 0.0 & & & & \\
\hline & & & 100 & 3.0 & 1.5 & & & & \\
\hline \multirow[t]{3}{*}{13} & ATS & TMP & 130 & 3.0 & 0.5 & 500 & 0.53 & 299 & 24 \\
\hline & & & 130 & 2.0 & 0.0 & & & & \\
\hline & & & 100 & 3.5 & 0.5 & & & & \\
\hline \multirow[t]{3}{*}{14} & ATS & TMP & 150 & 3.5 & 0.5 & 1420 & 0.41 & 314 & 42 \\
\hline & & & 190 & 2.5 & 4.5 & & & & \\
\hline & & & 100 & 3.5 & 0.5 & & & & \\
\hline \multirow[t]{3}{*}{15} & ATS & TMP & 150 & 3.5 & 0.5 & 1960 & 0.35 & 309 & 44 \\
\hline & & & 190 & 2.5 & 8.0 & & & & \\
\hline & & & 190 & 1.0 & 0.5 & & & & \\
\hline \multirow[t]{2}{*}{16} & ATS & TMP & 190 & 1.0 & 0.5 & 730 & 0.47 & 306 & 34 \\
\hline & & & 190 & 1.0 & 0.5 & & & & \\
\hline
\end{tabular}

The syntheses up to now described are of the one-pot type, the monomer and core being introduced simultaneously into the reactor in the quantities required to obtain the desired final polymer (in our case the fourth generation one). In the case of the preparation of some poly(bis-MBA) samples, in order to facilitate the regular growth of the branched chains and based on the different melting points of the core and the $\mathrm{AB}_{2}$-type monomer, a multi-step approach was adopted, as reported elsewhere for other hyperbranched polyesters [13-15]. According to this procedure, a small quantity of bis-MBA, which in the temperature conditions adopted is solid, was added to the reaction system containing TMP in the molten state. In this way the probability that a bis-MBA molecule reacts with the core should increase and consequently the growth of a regular hyperbranched structure should be favoured; the quantity of bis-MBA necessary to obtain the desired polymer was introduced in two successive additions. In this view, an initial temperature of $100-110{ }^{\circ} \mathrm{C}$ (at which only TMP is in the molten state) was chosen and the multi-step procedure was carried out for a series of 
syntheses (see the experimental part for the details), whose characteristics are reported in Table 3.

It has to be emphasized that such a procedure could not be applied using THEIC, its melting temperature being similar to that of bis-MBA. From the results reported in Table 3 it appears that rather high molecular weights were reached (see runs 14 and 15 ) and DB reached slightly higher values (at a fixed $M_{n}$ ) with respect to the one-pot syntheses.

Lastly, in run 16 the reaction temperature was raised to $190^{\circ} \mathrm{C}$ in all the three steps, but no significant difference in the polymer obtained was observed.

As in the literature no data are reported concerning hyperbranched poly(bis-MBA), the only significant comparison can be made with respect to bis-MPA based polymers, taking in mind the possible differences in the chemical structure, core moiety and generation number. Furthermore, as far as molecular weight is concerned, some difficulties arise from the fact that different analytical techniques are employed. For instance, many reported data have been obtained by SEC and, as well known, this technique fails in the case of hyperbranched polymeric samples, the values of molecular weight being in some cases much higher than the effective ones [7]. Notwithstanding this limitation, we can state that the maximum $M_{n}$ values reached by poly(bis-MBA) synthesized in our laboratories are similar to those reported for hyperbranched poly(bis-MPA), these last ranging from some hundreds to about 3000 $[7,16]$. In particular poly(bis-MPA) samples whose characteristics are reported in Ref. 7 are the most similar polymers to the poly(bis-MBA)s prepared by us, the core molecule employed being the same and pTSA being used as a catalyst. From the data there reported, it appears that the fourth generation polymer has a numberaverage molecular weight of 1799 (calculated from H-NMR data), which is very close to those of the poly(bis-MBA)s obtained in runs 2, 14 and 15. As concerns commercial samples, more detailed comparisons can hardly be made, chiefly due to the different functionality of the core molecule used.

As far as the DB values are concerned, there are similar to those reported in the case of other aliphatic hyperbranched polyesters $[11,14,16,17]$.

\section{Thermal properties}

Poly(bis-MBA) samples were afterwards examined by thermogravimetric analysis and differential scanning calorimetry. The investigation on the thermal stability was carried out both in air and under nitrogen atmosphere. From the thermogravimetric curves in air, the temperatures of initial decomposition $\left(T_{i d}\right)$ were determined and collected in Table 2 and 3. Similar results were obtained by means of TGA measurements under nitrogen atmosphere As can be seen, all the samples are characterized by a good thermal stability (up to about $290^{\circ} \mathrm{C}$ ), even though lower than that of poly(bis-MPA) [18]. Such a difference can be attributed to the presence in the monomeric unit of the polymers under investigation of an additional $\mathrm{CH}_{2}$ group. As regards calorimetric results, all the samples appeared to be completely amorphous, as evidenced by their calorimetric curves, which show only an endothermal baseline deviation associated to the glass transition phenomenon. The values of the glass transition temperature are collected in Table 2 and 3 and plotted in Figure 8 vs. molecular weight. 


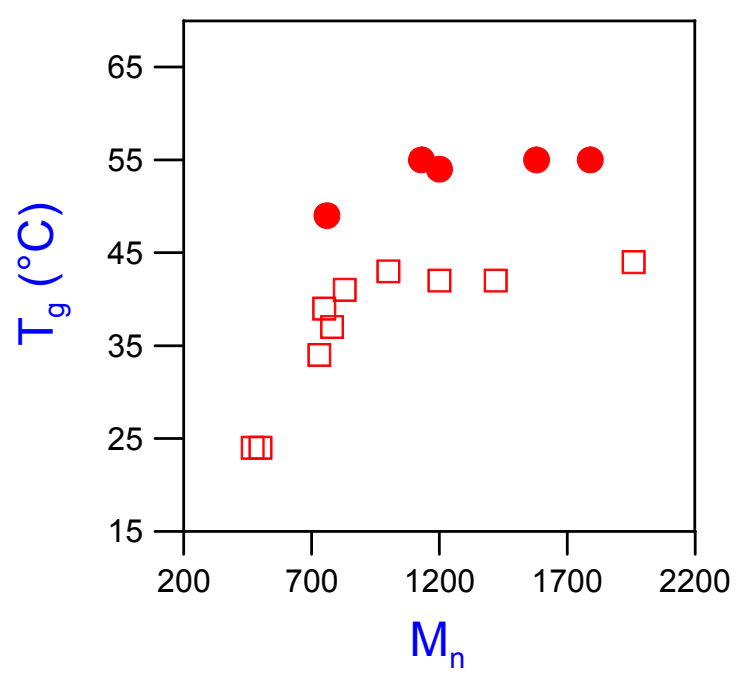

Fig. 8. Glass transition temperature vs. molecular weight for poly(bis-MBA) synthesized using (•) TFSc and $(\square)$ pTSA as catalysts.

From Fig. 8 different trends can be noted for samples obtained using different catalysts, even though in both cases the glass transition temperature levels off at $M_{n}$ near 1000. In particular, $\mathrm{T}_{\mathrm{g}}$ values appear to be significantly higher for the samples synthesized using TFSc. The trend of $\mathrm{T}_{\mathrm{g}}$ "leveling-off" with increasing molecular weight is characteristic of the hyperbranched structure and is rather similar to that observed for linear polymers [19], with some differences. Indeed, besides molecular weight, several factors may affect the glass transition temperature of a hyperbranched polymer, e.g. the content and the chemical characteristics of end groups, the kind of branching junctions, and the compactness of the macromolecular skeleton. Usually, $\mathrm{T}_{\mathrm{g}}$ decreases with the increment of the end group content, whereas it increases with increasing their polarity, the junction density and the compactness of the hyperbranched structure [20]. As far as the $T_{g}$ dependence on the content and the chemical characteristics of end groups is concerned, it can be noted that samples with similar molecular weights but obtained with different catalysts (see Table 2 and 3 ) are characterized by similar amounts of $\mathrm{OH}$ terminal groups and higher contents of $\mathrm{COOH}$ groups. As a consequence, polymers synthesized using pTSA should show a higher glass transition temperature because of the increase in the number of hydrogen bonds. Moreover, the results obtained indicate that other factors have to be considered, primarily the structure of the polymeric skeleton. In fact, as reported above, only when ScTF is used gelation sometimes occurs and this fact suggests that by employing such a catalyst a more disordered branched polymer originates, with a substantially different structure with respect to the regular one deriving from the 'perfect' polymerization of an $A B_{2}$ monomer. This remark could give the basis in order to explain the higher $\mathrm{T}_{\mathrm{g}}$ values of the hyperbranched samples obtained using ScTF as catalyst.

In addition, it is well known that in the synthesis of many kinds of hyperbranched polymers relevant amounts of cyclic molecules can form, whose plasticizing effect influences the glass transition phenomenon, leading to lower $T_{g} s$. In this view, further studies to determine the cyclic content in hyperbranched poly(bis-MBA), requiring rather sophisticated analytical techniques [7, 21], are in progress.

Lastly, it has to be stated that the $T_{g}$ values appear significantly higher than those reported for commercial or laboratory synthesized poly(bis-MPA) [16, 18]. These 
differences can be attributed to some factors, such as, lower contents of carboxylic end-groups and, in some cases, a different functionality core moiety for poly(bisMPA)s.

\section{Conclusions}

In this paper we have presented the results of a research regarding the synthesis and the characterization of a novel aliphatic hyperbranched polyester, based on 2,2bis(hydroxymethyl)butyric acid. The work done permitted to optimize the synthesis conditions, in terms of reaction temperature and time, catalyst employed, etc. With regard to catalyst, pTSA (which was previously used in the syntheses of several other hyperbranched polyesters) seemed to be the most efficient, gelation being avoided in all the conditions used and samples of relatively high molecular weight and degree of branching being obtained. The polymers synthesized showed in fact $M_{n}$ up to about 1800 , with a DB ranging from 0.32 to 0.53 , both similar to what reported in the case of other hyperbranched polyesters. Higher values of the glass transition temperature (which appeared to be dependent on the catalyst employed) can be attributed to the higher content of carboxylic end group.

\section{Experimental part}

\section{Reagents}

2,2-bis(hydroxymethyl)butyric acid (bis-MBA), 1,1,1-tris-(hydroxymethyl)-propane (TMP), 1,3,5-tris(2-hydroxyethyl)cyanuric acid (THEIC), p-toluensolfonic acid (pTSA), scandium trifluoromethanesulfonate (ScTF), titanium tetrabutoxide $\left(\mathrm{Ti}(\mathrm{OBu})_{4}\right)$, dibutyltin oxide, all from Aldrich, were used as supplied, except $\mathrm{Ti}(\mathrm{OBu})_{4}$, which was distilled before use.

\section{Synthesis of hyperbranched polymer samples}

Hyperbranched polymer samples were synthesized in bulk starting from 2,2bis(hydroxymethyl) butyric acid (an $\mathrm{AB}_{2}$-type monomer), using TMP or THEIC as core molecule and employing in most cases pTSA or ScTF as catalyst. In all the experiments a core/monomer molar ratio of 1/45 was used, corresponding to a fourth generation polymer. After some preliminary syntheses were carried out in order to optimize catalyst concentration, amounts of about 4.3 and $3.2 \mathrm{~g}$ of catalyst $/ \mathrm{kg}$ of monomer), corresponding to 0.35 and $0.10 \mathrm{~mol} \%$ concentrations, were employed in the case of pTSA and ScTF, respectively.

The syntheses were carried out in a $500 \mathrm{~mL}$ glass reactor, with a thermostatted silicon oil bath, temperature and torque being continuously recorded during polymerization, according to the usual two-stage polymerization procedure. In the first stage, at atmospheric pressure and under argon flow, the temperature was raised to $160-190{ }^{\circ} \mathrm{C}$ and maintained there until about $90 \%$ of water was distilled off. In the second stage, at the same temperature, the pressure was reduced to 10 mbar, in order to facilitate water removal.

As the slow addition of the $A B_{x}$ monomer could affect the characteristics of the resulting hyperbranched polymer, in terms of degree of branching and polydispersity, a set of multi-step reactions were carried out. In particular, in these cases the total prefixed amount of monomer was added into the reactor containing catalyst and core in three subsequent steps. The amount relative to the first addition was that 
necessary to obtain a second generation hyperbranched polymer, whereas the second and the third introductions were planned to obtain a third and fourth generation polymer, respectively. Each monomer addition was followed by a $1^{\text {st }}$ stage at atmospheric pressure under Argon flow and by a $2^{\text {nd }}$ stage under reduced pressure.

\section{NMR spectroscopy}

The ${ }^{1} \mathrm{H}$ and ${ }^{13} \mathrm{C}-\mathrm{NMR}$ spectra were recorded on a Varian XL-400 instrument using DMSO- $d_{6}$ as a solvent and tetramethylsilane (TMS) as an internal reference. ${ }^{1} \mathrm{H}-\mathrm{NMR}$ spectra were recorded at room temperature for solutions with a polymer concentration of $0.5 \mathrm{wt} \%$ (a relaxation delay of $0 \mathrm{~s}$, an acquisition time of $1 \mathrm{~s}$ and up to 100 repetitions). ${ }^{13} \mathrm{C}-\mathrm{NMR}$ spectra were obtained using $10 \mathrm{wt} \%$ solutions and a full decoupling mode with a NOE effect (a relaxation delay of $1 \mathrm{~s}$, an acquisition time of 1 $s$ and up to 700 repetitions). Different measurement conditions is described in the text where needed.

\section{End-group analysis}

Hydroxyl end-group content was determined following a procedure reported in literature [22]: a sample of about $0.6 \mathrm{~g}$ was dissolved in $10 \mathrm{~mL}$ of acetylation mixture (freshly prepared by mixing one part of acetic anhydride with 9 parts of pyridine) under heating at reflux for 60 minutes. When the sample was completely dissolved and the solution cooled to room temperature, the condenser was washed with $20 \mathrm{~mL}$ of acetone, before flask removal. Water $(20 \mathrm{~mL})$ and a few drops of phenolphthalein indicator were added. The solution was titrated with a $\mathrm{NaOH}(1.00 \mathrm{M})$ solution, under stirring until a pink colour appears and remains for five seconds, being $V_{2}$ the titrimetric volume. Blank tests were performed with the same procedure and the corresponding titrimetric volume was indicated as $V_{1}$. The hydroxyl number was calculated as $\left(\mathrm{V}_{1}-\mathrm{V}_{2}\right) \mathrm{C} / \mathrm{w}$ (in $\mathrm{mmol} / \mathrm{g}$ of polymer), where $\mathrm{C}$ is molar concentration of $\mathrm{NaOH}$ and $\mathrm{w}$ is the mass of the sample in $\mathrm{g}$.

As concerns carboxylic end-group content, samples of $1 \mathrm{~g}$ were dissolved in $20 \mathrm{~mL}$ of acetone and titrated with a $\mathrm{NaOH}(1.00 \mathrm{M})$ solution, using phenolphthalein as indicator. Blank test were performed on pure solvent mixture.

The values of end-group contents, reported in Table 2, are the averages of several replicates.

\section{Thermal analysis}

Thermogravimetric analysis was performed both in air and under nitrogen atmosphere using a Perkin Elmer TGA7 apparatus (gas flow: $50 \mathrm{~mL} / \mathrm{min}$ ) at 10 ${ }^{\circ} \mathrm{C} /$ min heating rate up to $900^{\circ} \mathrm{C}$.

Calorimetric measurements were carried out by means of a Perkin Elmer DSC7 instrument equipped with a liquid sub ambient accessory and calibrated with high purity standards (indium and cyclohexane). The external block temperature control was set at $-60{ }^{\circ} \mathrm{C}$. Weighed samples (c.a. $10 \mathrm{mg}$ ) were encapsulated in aluminium pans and heated to $100^{\circ} \mathrm{C}$ at a rate of $20^{\circ} \mathrm{C} / \mathrm{min}$ (first scan), held there for $1 \mathrm{~min}$, and then rapidly quenched to $-30{ }^{\circ} \mathrm{C}$. Finally, they were reheated from $-30{ }^{\circ} \mathrm{C}$ to $100{ }^{\circ} \mathrm{C}$ at a heating rate of $20{ }^{\circ} \mathrm{C} / \mathrm{min}$ (second scan). The glass-transition temperature $\mathrm{T}_{\mathrm{g}}$ was taken as the midpoint of the heat capacity increment $\Delta c_{p}$ associated with the glassto-rubber transition. The specific heat increment $\Delta \mathrm{c}_{p}$, associated with the glass 
transition of the amorphous phase, was calculated from the vertical distance between the two extrapolated baselines at the glass transition temperature.

\section{References}

[1] Hult, A.; Johansson, M.; Malstrŏm, E. Adv. Polym. Sci. 1997, 143, 1.

[2] Voit, B. J. Polym. Sci, Polym. Phys. Ed. 2000, 38, 2505.

[3] Yates, C. R.; Hayes, W. Eur. Polym. J. 2004, 40, 1257.

[4] Gottschalk, C.; Frey H. Macromolecules 2006, 39(5), 1719.

[5] Smet, M.; Gottschalk, C.; Skaria, S.; Frey H. Macromol. Chem. Phys. 2005, 206(24), 2421.

[6] Kricheldorf, H. R.; Stöber, O.; Lubbers, D. Macromolecules 1995, 28, 2118.

[7] Burgath, A.; Sunder, A.; Frey, H. Macromol. Chem. Phys. 2000, 201, 782.

[8] Žagar, E.; Žigon, M. J. Chroma. A 2004, 1034, 77.

[9] Žagar, E.; Žigon, M. Macromolecules 2002, 35, 9913.

[10] Hawker, C. J.; Lee, R.; Fréchet, J. M. J. J. Am. Chem. Soc. 1991, 113, 4583.

[11] Komber, H.; Ziemer, A.; Voit, B. Macromolecules 2002, 35, 3514.

[12] Abdelrehim, M.; Komber, H.; Langenwalter, J.; Voit, B.; Bruchmann, B. J. Polym.

Sci., Part A: Polym. Chem. 2004, 42, 3062.

[13] Hanselmann, R.; Hölter, D.; Frey, H. Macromolecules 1998, 31, 3790.

[14] Magnusson, H.; Malmström, E.; Hult, A. Macromolecules 2000, 33, 3099.

[15] Cheng, K. C. Polymer 2003, 44, 1259.

[16] Žagar, E.; Žigon, M.; Podzimek, S. Polymer 2006, 47, 166.

[17] Jang, J ; Hak Oh, J. Polymer 1999, 40, 5985.

[18] Malmström, E.; Johansson M. ; Hult, A. Macromolecules 1995, 28, 1698.

[19] Wooley, K. L.; Hawker, C. J.; Pochan J. M.; Fréchet, J. M. J. Macromolecules 1993, 26, 1514.

[20] Liu, H.; Wilén, C. E. J. Polym. Sci., Part B: Polym. Phys. 2004, 42, 1235.

[21] Sahota, H. S.; Lloyd, P. M.; Yeates, S. G.; Derrick, P. J.; Taylor, P. C.; Haddleton, D. M. J. Chem. Soc., Chem. Commun. 1994, 21, 2445.

[22] Gao, F.; Schricker, S. R.; Tong, Y.; Culbertson, B. M. J. Macromol. Sci. - Pure Appl. Chem. 2002, A39(4), 267. 